\title{
Thyroid autoimmunity
}

\author{
Basil Rapoport and Sandra M. McLachlan \\ Autoimmune Disease Unit, Cedars-Sinai Research Institute and School of Medicine, University of California, Los Angeles, California, USA \\ Address correspondence to: Basil Rapoport, Cedars-Sinai Medical Center, 8700 Beverly Boulevard, Suite B-131, \\ Los Angeles, California 90048, USA. Phone: (310) 423-4774; Fax: (310) 423-0221; E-mail: rapoportb@cshs.org. \\ J. Clin. Invest. 108:1253-1259 (2001). DOI:10.1172/JCI200114321.
}

The seminal discovery in 1956 that Hashimoto thyroiditis (HT) is an autoimmune disease spelled the end of the concept of horror autotoxicus and introduced the reality of human autoimmunity. In the same year, Graves disease (GD) was discovered to be directly caused by a serum factor, subsequently identified as an autoantibody. Perhaps because of relatively effective treatment and the absence of a strong financial reason for pharmaceutical companies to develop a cure, GD and HT, the most common organ-specific diseases affecting humans, are not targeted for intensive investigation. Nevertheless, these diseases have served as invaluable models for understanding the pathogenesis of organ-specific autoimmunity in general. Whereas autoimmune thyroiditis occurs spontaneously in some susceptible animals (rodents and chickens), there is no spontaneous animal model of GD. Induced animal models of autoimmune thyroiditis have been available for many years. More recently, antibody-mediated hyperthyroidism has also been induced in rodents by a number of imaginative approaches (reviewed in ref. 1). However, in this Perspective, we will focus on human disease, which, although more difficult to study than animal models, is likely to generate information more relevant to human pathology.

\section{Autoantigens in autoimmune thyroid disease}

The study of HT and GD has been facilitated by the identification, molecular cloning, and expression of dominant and specific target antigens, thyroid peroxidase (TPO; reviewed in ref. 2) and the thyrotropin receptor (TSHR; reviewed in ref. 3). TPO, the primary enzyme involved in thyroid hormonogenesis, was initially identified in 1959 as the "thyroid microsomal antigen." As discussed below, it is uncertain whether TPO autoantibodies or TPO-specific T cells are the primary cause of thyroid inflammation, which can lead, in some individuals, to thyroid failure and hypothyroidism. On the other hand, GD is unquestionably caused by a humoral response to the TSHR. Autoantibodies mimic the action of the ligand TSH, thus activating the TSHR and directly causing hyperthyroidism. The autoimmune response to thyroglobulin, the most abundant thyroid protein, appears to play a lesser role in human thyroid autoimmunity than in animal models of thyroiditis. Similarly, although the recent molecular cloning of the thyroid sodium-iodide symporter (4) has created a flurry of interest in its potential role as an autoantigen, emerging evidence does not support this likelihood.

The molecular cloning of TPO led to the surprising realization that this enzyme is a cell surface protein (reviewed in ref. 2). TPO is a $107-\mathrm{kDa}, 933-$ amino acid residue glycoprotein with a single membrane-spanning segment and is present as a dimer on the apical surface of the thyroid follicular cell. A stop codon introduced at the TPO ectodomain-plasma membrane junction converts the 933-amino acid membrane-associated molecule into an 845-residue secreted protein that can be

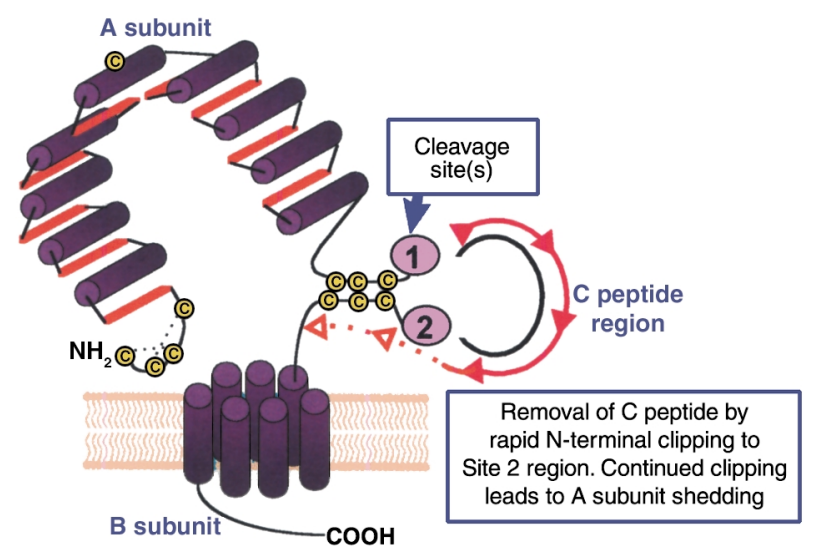

Figure 1

Schematic representation of the TSHR with its large (397-amino acid residue without signal peptide) ectodomain, seven membrane-spanning segments, and short cytoplasmic tail. TSHR intramolecular cleavage into $A$ and $B$ subunits is associated with the loss of a $C$ peptide region that corresponds approximately to a 50-amino acid "insertion" in the TSHR absent in the noncleaving $\mathrm{LH}$ and $\mathrm{FSH}$ receptors. The $\mathrm{C}$ peptide region is not removed intact. Following cleavage at upstream Site 1 , the $C$ peptide is rapidly degraded downstream to the Site 2 region. Evidence suggests that $\mathrm{N}$-terminal degradation of the $\mathrm{B}$ subunit continues thereafter, leading to loss of the Cys residues tethering the $A$ subunit and to shedding of the latter. The Cys-rich N-terminus of the A subunit is an important component of thyroid-stimulating autoantibodies and is likely to contain two disulfide bonds (hypothetically shown by dotted lines) contributing to a conformationally important portion of the molecule. 


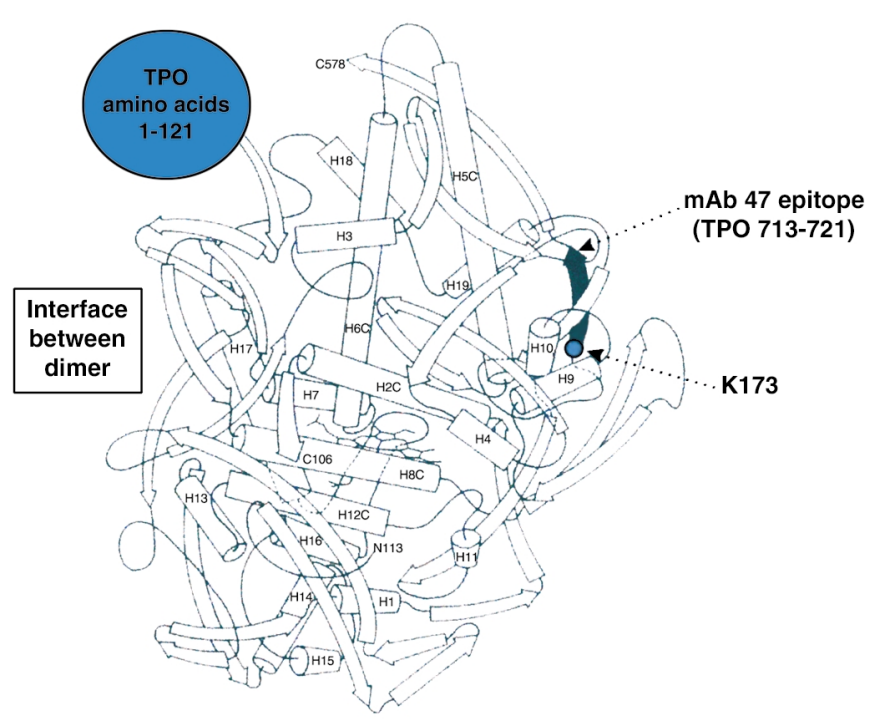

\begin{abstract}
Figure 1
Hypothetical three-dimensional structure of the TPO ectodomain based on the structure of myeloperoxidase (MPO). The ribbon diagram represents one TPO monomer beginning at residue 122 because no structural information is available on the $\mathrm{N}$-terminal 121 amino acid prosequence of MPO (large blue circle), which is removed from the mature molecule. The C-terminus of MPO ("C578") corresponds to TPO amino acid residue 736. Therefore, the diagram also does not provide insight into the region of the TPO ectodomain extending downstream to its insertion into the plasma membrane (residue 848). Epitopic footprinting identified lysine (K) 713 to be within the epitope of one of the human monoclonal TPO autoantibodies (TR1.9) that define the TPO immunodominant region. This residue is included in the linear epitope (shaded) of mouse mAb no. 47 , which is recognized by a small proportion of TPO autoantibodies in an individual patient's serum (16). Modified, with permission, from refs. 5, 19 (copyright 2001, The American Association of Immunologists).
\end{abstract}

purified in milligram amounts from medium conditioned by transfected mammalian or insect cells. Patients' autoantibodies recognize the TPO ectodomain to the same extent as the holoenzyme. Although small crystals have been obtained from purified TPO, these crystals have not, as yet, provided $\mathrm{x}$-ray diffraction data of sufficient resolution to elucidate the three-dimensional structure of the molecule. Nevertheless, a reasonable image of the TPO ectodomain can be predicted from the crystal data for myeloperoxidase (5) (Figure 1), a closely related molecule with relatively uniform amino acid homology (about 47\%).

The TSHR, a member of the G protein-coupled receptor family with seven membrane-spanning segments, is closely related to the receptors for the other glycoprotein hormones, luteinizing hormone (LH) and follicle-stimulating hormone (FSH) (reviewed in ref. 3). Even before its molecular cloning, the TSHR was known to contain two subunits, an extracellular A subunit and a largely transmembrane B subunit, linked by disulfide bonds (6). Translation of both subunits from a single mRNA species indicated that the TSHR forms by intramolecular cleavage from a larger precursor. Cleavage occurs in the mature receptor after it reaches the cell surface (7). Recently, TSHR cleavage into $A$ and $B$ subunits has been found to be associated with the loss of an intervening $C$ peptide segment corresponding approximately to a 50-amino acid "insertion" uniquely present in the TSHR and not present in the noncleaving $\mathrm{LH}$ and FSH receptors (8). The C peptide does not appear to be released intact but is likely to be removed in small segments after cleavage at upstream Site 1 terminating at downstream Site 2 (Figure 2). The precise TSHR cleavage sites have not been identified because the unknown enzyme responsible for cleavage, perhaps a membrane-associated matrix metalloproeinase, does not require a specific amino acid motif. Of potential importance in the immune response to the TSHR, most A subunits are shed from the surface following cleavage, at least in cultured cells (9). Shedding is suggested to involve dissolution by protein disulfide isomerase of the disulfide bonds tethering the A and B subunits. Alternatively, there is evidence that following rapid removal of the $\mathrm{C}$ peptide region, slower progression of the cleavage process removes the critical cysteines at the $\mathrm{N}$-terminus of the B subunit (Figure 2).

Obtaining TSHR antigen for diagnostic and investigative purposes has been much more difficult than for TPO. The TSHR is a labile molecule with a highly conformational structure, and only small amounts can be purified from human thyroid tissue. When expressed in prokaryotic cells, yeast, or insect cells, both the TSHR holoreceptor and its ectodomain are largely insoluble and cannot be recognized by autoantibodies, even after refolding. Mammalian cells are required for effective autoantigen expression. Unlike TPO, truncation of the TSHR at its insertion in the plasma membrane leads to the intracellular retention of an immature ectodomain, which is poorly recognized by autoantibodies. Recent work shows that when it is truncated further upstream, in the vicinity of upstream cleavage Site 1 , a TSHR ectodomain module is secreted that corresponds approximately to the A subunit (10). This antigen, containing $40 \%$ of its mass as N-linked glycan, can be purified in large amounts and is capable in nanogram amounts of neutralizing autoantibodies in Graves patients' sera. Other successful approaches to producing suitable autoantigen include fusing the entire TSHR ectodomain to a glycosylphosphatidylinositol (GPI) anchor or to a cleavable CD8 fusion protein. To our knowledge, crystals of TSHR ectodomain have not been obtained and its three-dimensional structure is presently unknown. However, the more conserved midportion of the TSHR ectodomain, containing nine leucine-rich repeats (LRRs), has been modeled based on the known structure of LRRs in ribonuclease A inhibitor (11).

\section{Autoantibodies}

The precise epitopes and immunoglobulin gene usage of autoantibodies can only be obtained by the molecular cloning of human autoantibodies. Among the organ-specific autoimmune diseases affecting humans, this goal has been met best in the case of TPO autoan- 
tibodies $(12,13)$. Besides providing highly sensitive and specific assays for serum autoantibodies, recombinant, conformationally intact TPO has allowed for the molecular cloning and characterization of an extensive repertoire of nearly 200 human TPO autoantibodies. With few exceptions, these autoantibodies have been isolated from immunoglobulin gene combinatorial libraries constructed from thyroid-infiltrating B (probably plasma) cells. The high frequency of TPO autoantibodies in these libraries is consistent with previous evidence for the importance of thyroid lymphocytes as a source of TPO autoantibodies (14). These autoantibodies, the sine qua non of autoimmune thyroiditis, are also present in serum at concentrations in the microgram- to milligram-per-milliliter range.

Whereas the individual $\mathrm{H}$ and $\mathrm{L}$ chains of TPO autoantibodies isolated by the combinatorial library approach are unquestionably those that occur in human disease in vivo, it is controversial whether the heavy $(\mathrm{H})$ and light $(\mathrm{L})$ chain pairings seen in these experiments reflect the true structure of autoantibodies formed in vivo. Nonetheless, $\mathrm{H}$ and $\mathrm{L}$ chain shuffling experiments (12) strongly favor this interpretation. In addition, these recombinant immunoglobulins closely resemble serum autoantibodies with respect to their very high affinities $\left(10^{-10} \mathrm{M} \mathrm{Kd}\right.$ range $)$ and the epitopes they recognize. The independent isolation of similar genes and pairings from thyroid glands obtained on different continents further support the notion of correct $\mathrm{H}+\mathrm{L}$ pairing. The germline genes used by TPO autoantibody $\mathrm{H}$ and $\mathrm{L}$ chains are not different from those of many other antibodies. However, the $\mathrm{H}$ chain genes are remarkable for their high degree of somatic mutation, consistent with the high affinity of the antibodies and suggesting an antigen-driven affinity maturation process. Another feature is the recurrence of certain germline genes among many TPO autoantibodies isolated from at least seven patients. In addition, there is evidence that use of a particular $\mathrm{Vk}$ light chain gene (02/012) contributes to the epitopic domain recognized by TPO autoantibodies.

The cloning of human TSHR autoantibodies has proven a much more difficult undertaking. Although there are numerous reports over the past decade on the isolation of such autoantibodies by conventional cell fusion or immortalization approaches, none of these clones fulfills the criteria expected of such autoantibodies (15). A number of factors have contributed to this difficulty. TSHR autoantibodies in serum are typically present at concentrations in the nanogram-permilliliter range (10), two to three orders of magnitude lower than that of TPO autoantibodies. As described above, purification of conformationally intact, recombinant TSHR has only recently been attained, and this material is inherently unstable. Finally, perhaps because of its very high carbohydrate content, the TSHR ectodomain is a "sticky" molecule that can bind nonspecifically to IgG in normal sera. We do not subscribe to the view that a proposed low affinity of these autoantibodies for antigen or a requirement for antigen dimerization represents a major impediment to isolating human TSHR autoantibodies. Serum TSHR autoantibodies can be readily neutralized using nanogram concentrations of monomeric TSHR ectodomain.

The low TSHR autoantibody concentration in serum is consistent with previous observations of restricted $\mathrm{L}$ chain usage and limitation to the IgG1 subclass, which suggested that these autoantibodies are oligoclonal (reviewed in ref. 3). This difference in concentration and clonality of TSHR and TPO autoantibodies may be of pathophysiological significance. Potent and functional TSHR autoantibodies can cause clinical symptoms at a very early stage of the autoimmune response. In contrast, there is much greater tolerance for thyroid cell damage (whether humoral or cell-mediated) because of the regenerative capacity of the thyroid under the influence of TSH Therefore, unlike type 1 diabetes, where pancreatic islet $\beta$ cells lack a compensatory tropic hormone, smoldering thyroiditis can exist for decades before glandular failure and the onset of clinical symptoms.

\section{B cell epitopes}

Information on the epitopes of autoantibodies may provide valuable insight into the nature of the autoimmune response to the thyroid. Much more is known regarding TPO autoantibodies than TSHR autoantibodies, mainly because of the success in the molecular cloning and expression of TPO autoantibody genes. Autoantibodies are highly sensitive to TPO conformation and generally fail to recognize the denatured molecule, synthetic peptides with TPO sequences, or recombinant TPO expressed in prokaryotes. These antibodies do not bind the glycan component of TPO, but bind solely to polypeptide epitopes (reviewed in ref. 12).

Early studies mapping TPO autoantibody epitopes with murine monoclonal antibodies established that autoantibodies recognize a restricted region on TPO (16). Competition studies using recombinant human TPO autoantibodies (expressed as Fab's) confirmed the epitopic restriction of serum TPO autoantibodies and helped define four closely associated, largely overlapping epitopic domains for these TPO autoantibodies (17). The ability of a pool of four Fab's to these epitopes to inhibit the binding to TPO of most TPO autoantibodies in an individual patient's serum, indicates that these epitopes comprise the immunodominant region of the protein.

Quantitative competition studies with these four recombinant autoantibody Fab's have also been used to characterize the polyclonal TPO autoantibodies in patients' sera (18). Analysis of such TPO autoantibody epitopic "fingerprints" (12) has been informative in several respects. First, there is no relationship between this fingerprint and the likelihood of a patient with HT to develop thyroid failure. Second, the epitopic fingerprint in an individual patient is unaltered during pregnancy and the postpartum period despite hormonal influences that lead to large changes in TPO autoantibody titer. Third, fingerprints remain essentially unchanged even over periods as long as 13 years. Thus, there is no evidence for epitope spreading over time. 
Finally, segregation analysis studies on multiplex families with TPO autoantibodies suggest a genetic component to the TPO autoantibody fingerprints.

It is also noteworthy that autoantibodies to the TPO immunodominant region do not inhibit TPO enzymatic activity. Therefore, if these autoantibodies contribute to thyroid inflammation, rather than simply being markers of disease, they are not directly responsible for the development of hypothyroidism.

Several approaches have been taken to localize the immunodominant region on TPO, including autoantibody recognition of TPO polypeptide fragments or TPO-myeloperoxidase chimeric molecules, and competition for autoantibody binding by polyclonal rabbit antisera to defined synthetic peptides. These efforts have produced widely differing results, none of which are definitive. Possible reasons for this uncertainty include the conformational nature of the epitopes in this region and the fact that recognition of a polypeptide fragment by polyclonal serum could reflect a minority of TPO autoantibodies that bind to epitopes outside the immunodominant region.

Clearly, the human monoclonal Fab's that define the TPO immunodominant region are the optimum reagents to define precisely the exact location of the immunodominant region. Recently, one of these Fab's has been used in an epitopic footprinting technique involving protection from biotinylation of surface lysine residues. Lysine 731 has been identified and provides a beacon for future mutagenesis studies (Figure 1) (19). The ultimate resolution of these questions will come with the determination of crystal structures for TPO-monoclonal autoantibody complexes. At present, one of these monoclonal autoantibody Fab's has been crystallized alone and its three-dimensional structure elucidated (20).

Because of the functional nature of TSHR autoantibodies, determination of their precise epitopes is even more important than for TPO autoantibodies and could have important implications for future therapeutic strategies in GD. However, identification of amino acids on the TSHR with which autoantibodies interact has been difficult, for a number of reasons. First, the requirement for conformationally intact antigen is even more stringent for TSHR autoantibodies than for TPO autoantibodies. Evidence from TSHRgonadotropin hormone chimeric receptors suggests that TSHR autoantibody epitopes are discontinuous (21). Second, autoantibodies to the TSHR autoantibodies, unlike those for TPO, do not recognize the deglycosylated, or poorly glycosylated, form of the antigen. Whether the extensive glycan component of the TSHR ectodomain contributes to autoantibody epitopes is unknown, since reduced glycosylation could occur secondary to TSHR malfolding and failure to traffic to the cell surface (22). Third, no high-affinity, functional human monoclonal autoantibodies are available to the TSHR. Finally, a variety of types of TSHR autoantibodies can occur in patients' sera: thyroid stimulatory antibodies (TSAb) that activate the TSHR and TSHblocking antibodies (TBAb) that inhibit the binding of
TSH with activating the TSHR. Evidence also exists for "neutral" TSHR autoantibodies that neither stimulate nor block ligand binding.

In studies of TSHR mutants and chimeric receptors, it has been observed that the $\mathrm{N}$-terminus and C-terminus of the TSHR ectodomain are critical for TSAb and TBAb function, respectively $(21 ; 23)$. Moreover, a number of TSHR amino acids have been implicated in autoantibody binding. However, there is a prevalent misconception that TSAb and TBAb bind only at the $\mathrm{N}$ - or C-terminus. Most likely, although the epitopes for TSAb and TBAb diverge the most at the TSHR $\mathrm{N}$-terminus, there is epitopic overlap between these autoantbodies at more downstream TSHR locations, as would be anticipated for discontinuous epitopes.

$T$ cell epitopes, $T$ cell receptor $V$ genes, and cytokines

Production of IgG class autoantibodies requires "help" from $\mathrm{CD} 4^{+} \mathrm{T}$ cells. There are numerous studies on $\mathrm{T}$ cell responses to TPO and TSHR synthetic peptides using lymphocytes from peripheral blood and, to a lesser extent, from thyroid and lymph nodes. Although certain regions of TPO and the TSHR have been observed to be more active than others, variable responses are seen to multiple peptides, encompassing most of the TPO and TSHR molecules. Further, the T cell proliferative responses to TPO or TSHR synthetic peptides are usually small and, in some cases, there are no differences between the responses in patients and in controls. These data suggest considerable $T$ cell epitope diversity in the patient population and create difficulties for therapeutic strategies involving TPO peptides.

Because the thyroid is enriched in thyroid-specific $\mathrm{T}$ and $\mathrm{B}$ cells, there was a recent period of interest in comparing the $\mathrm{V}$ gene repertoire of $\mathrm{T}$ cell receptors in thyroid tissue and peripheral blood from patients with autoimmune thyroid disease. However, such analyses have yielded controversial results (reviewed in ref. 12). Restriction of $T$ cell receptor $V \alpha$ (but not $V \beta$ ) has been observed in some, but not all, thyroids from patients with thyroid autoimmunity. Moreover, regardless of whether intrathyroid $\mathrm{T}$ cell receptors are oligoclonal, this approach cannot address the issue of $\mathrm{T}$ cell antigen specificity, because intrathyroidal $\mathrm{T}$ cells respond to a variety of nonthyroidal antigens, as well as to different thyroid autoantigens. In addition, it is possible that restricted $T$ cell receptor $V$ region usage may not always correspond to identical epitopic recognition. Thus, clones using identical $T$ cell receptors, encoded by identical $V \beta$ and $V \alpha$ genes and differing only in their joining (Ja) regions, recognize different, nonoverlapping epitopes of TPO (24).

The cytokine profiles of TSHR- or TPO-specific T cell clones, together with flow cytometry data for in vivo activated thyroid-infiltrating T cells and RT-PCR analysis of thyroid tissues, suggest that IFN- $\gamma$ predominates in autoimmunity associated with thyroid cell damage (HT), whereas IL-4 is more evident in autoantibodymediated disease (GD) (reviewed in ref. 12). However, both GD and HT patients have IgG4 and IgE TPO 
autoantibodies, isotypes for which IL-4 is a switch factor. Conventional wisdom holds that autoimmune diseases can be treated by immune deviation from a Th1 to a Th2 response. Although the association of Th1 responses with HT, on the one hand, and of Th2 responses with GD, on the other, may not be clear-cut, immune deviation toward Th2 may be detrimental in autoimmune thyroid disease.

\section{Presentation of endogenously processed} thyroid antigens

Given the rather disappointing findings thus far in characterizing $\mathrm{T}$ cells with synthetic peptides, future prospects seem brighter in studies in which TPO or TSHR T cell epitopes are presented by cells that themselves process the antigen. Of the professional antigenpresenting cells, immortalized $\mathrm{B}$ cells are technically the most practical to study in humans. However, although such B cells can present peptide-MHC complexes, their capacity to internalize intact exogenous antigen for processing is limited. This handicap has been overcome by stably transfecting Epstein-Barr virus-immortalized $B$ cells (EBVLs) with the cDNA for TPO or the TSHR (reviewed in ref. 12). This approach has recently been used to generate nonclonal $\mathrm{T}$ cell lines as well as to study previously isolated $\mathrm{T}$ cell clones $(25 ; 26)$. Extension of this approach to more patients and with cloned $\mathrm{T}$ cells may provide important new information. A crucial issue for future investigation is the more meaningful, but more difficult, readout of $\mathrm{T}$ cell function in terms of the ability to induce functional autoantibody synthesis by autologous B cells. Such studies are particularly important in $\mathrm{GD}$, in which TSAb's are the direct cause of disease.

Of even greater pathophysiological relevance than stably transfected EBVLs will be antigen presentation by cells that use surface receptors to capture, internalize, and process exogenous antigen. With regard to thyroid autoimmunity, at least, such studies are still in their early stages (27). Macrophages and, in particular, dendritic cells have the greatest capacity for spontaneous, nonspecific capture and uptake of antigen. On the other hand, B cells, by virtue of their specific antigen receptors, can capture and present specific antigen present at very low concentration in the surrounding milieu, thereby perpetuating and amplifying $\mathrm{T}$ cell responses. Moreover, antibodies complexed to antigen can modulate antigen processing and enhance or suppress presentation of different $\mathrm{T}$ cell determinants (28). The importance of $\mathrm{B}$ cells in antigen presentation is increasingly being recognized in a variety of immune responses to infections and parasites, as well as in autoimmunity. Thus, diabetes does not develop in NOD mice in the absence of $\mathrm{B}$ cells, probably because $\mathrm{B}$ cells are required to present islet-cell autoantigens to $\mathrm{T}$ cells (see, for example, ref. 29. Overall, an important direction for future investigation is to determine whether thyroid autoantibodies, besides directly causing thyrotoxicosis (and possibly thyroiditis), have an additional role, either as secreted molecules or as membrane receptors on B cells, in presenting thyroid autoantigens to $\mathrm{T}$ cells in human thyroid autoimmunity.
There has also been a resurgence of interest in antigen presentation by "nonprofessional" cells, a phenomenon first recognized in autoimmune thyroid disease in which thyrocytes were observed to "aberrantly" express MHC class II molecules and to function as antigen-presenting cells (30). These observations provided the impetus for studies involving presentation of TPO and the TSHR by stably transfected immortalized $\mathrm{B}$ cells (see above). Recently, further support for the concept of aberrant MHC class II expression has been the development of a model of GD by injecting mice with fibroblasts coexpressing syngeneic MHC class II and the TSHR (31). Similarly, mice injected with fibroblasts coexpressing class II and TPO, but not with TPO and adjuvant, develop TPO antibodies, and this humoral response resembles that of patients with respect to autoantibody affinity and epitope restriction to the immunodominant region (32).

Mechanisms of thyroid cell damage

The mechanism of thyroid cell damage in HT, long an important unanswered question, recently re-emerged as a focus of interest with the report that thyroid cells constitutively express Fas ligand (FasL). Cytokineinduced Fas expression on the same cells has therefore been suggested to be the primary cause of thyroid cell death (33). These findings have been controversial (reviewed in ref. 34), but this important issue will probably be resolved in the next few years. Regardless of whether thyrocyte expression of Fas or FasL is induced by cytokines, the fundamental question is why these cytokines are produced in the thyroid in the first place. Only a mechanism involving the adaptive immune response to specific thyroid antigens can explain the occurrence of thyroiditis.

It should also not be overlooked that $\mathrm{T}$ cell-mediated and antibody-dependent cellular cytotoxicity induced by TPO autoantibodies can contribute to thyroid cell killing (reviewed in ref. 12). Apoptosis may well play a significant role in the final pathway of thyroid cell destruction but cannot explain the basic mechanism of disease. Studies of Fas-FasL interactions should not, therefore, divert the pursuit of antigen-specific mechanisms of thyroid damage.

\section{Genetics}

A major effort is presently underway on different continents to identify the genes that contribute to the pathogenesis of GD and HT (reviewed in ref. 35). Whole-genome scanning has identified several loci, but the lod scores are relatively low and confirmation of these loci by multiple groups is still lacking . Among candidate genes, MHC class II antigens are associated with clinical disease but the relative risks are low, and polymorphisms of the TSHR and TPO have been excluded by linkage analysis. Only CTLA4 has generated sustained interest. In our view, a CTLA4 polymorphism could influence the amplitude of the immune response and contribute to autoimmunity in general but cannot account for the antigen-specific nature of GD and HT. 
Recently, ascertainment in genetic studies of thyroid autoimmunity has shifted from overt disease toward more limited but more readily characterized phenotypes such as the presence of autoantibodies. TPO autoantibodies, in particular, are markers for subclinical disease, and previous evidence suggests a role for heredity in their development and epitopic characteristics (12). Clearly, the pursuit of genes is vitally important and will provide invaluable information in the future. However, autoimmune thyroid diseases are likely caused by multiple, relatively minor genes intertwined with environmental factors. Nongenetic factors, such as alterations in iodine intake and, perhaps, immune responses to microorganisms, should not be overlooked.

\section{Extrathyroidal manifestations}

The most enigmatic aspect of GD is why some patients develop orbital infiltrative disease that can also be a threat to vision. Less commonly, but always in association with Graves ophthalmopathy, infiltrative dermopathy may also occur. Despite early suspicions of a role for TSHR autoantibodies in the extrathyroidal manifestations of GD, many subsequent studies failed to show a relationship between TSHR autoantibody titer and ophthalmopathy. Consequently, other causes have been sought for the extrathyroidal manifestations of GD, including cell-mediated effector mechanisms, perhaps involving other cross-reacting antigens between the thyroid and orbital connective tissue. In recent years, accumulating evidence points toward induction by unknown factors of TSHR protein expression in orbital fibroblastic preadipocytes as a cause of ophthalmopathy (reviewed in ref. 36). One potentially unifying hypothesis for GD and its extrathyroidal manifestations (37) rests on the observation of constitutive TSHR expression in normal individuals at sites other than those classically affected in GD, and on evidence that a low-grade systemic, connective tissue inflammation occurs in GD. It has been proposed that the extent of this extrathyroidal inflammation is proportionate to the autoimmune response to the TSHR. Such inflammation, usually subclinical, is necessary but not sufficient for the development of ophthalmopathy or dermopathy. Additional local factors, such as gravitational dependency, trauma, cigarette smoking, and (most important) the anatomical constraints of the bony orbit, lead to the development of overt disease at specific sites.

Because present treatment of these distressing conditions is suboptimal and is not directed at the root cause, better understanding of Graves ophthalmopathy and dermopathy is urgently needed. Questions such as the possible existence of yet-to-be-recognized autoantigen(s) in orbital tissue need to be answered. On the other hand, if the autoimmune response to the TSHR is found to be directly involved in the pathogenesis of these disorders, efforts to reduce this response (as reflected in the TSHR autoantibody titer) may be of value. The thyroid is a major site of autoantibody production, and thyroid cells in HT and GD patients (but not in normal individuals) express MHC class II. Con- sequently, total ablation of thyroid tissue (a form of therapy advocated as successful three decades ago but now abandoned) may be therapeutically useful.

\section{Future directions}

Present therapies for GD and $\mathrm{HT}$, such as radioiodine or l-thyroxine administration, are not curative in that they do not reverse the underlying, fundamental pathogenesis. More effective avenues aimed at re-established immune tolerance to thyroid antigens will require a better understanding of the molecules involved. The present major emphasis on gene discovery in autoimmune thyroid diseases, while essential, is unlikely to produce all the answers. Similarly, the current focus on apoptosis as a cause of thyroid failure can provide only limited information.

In our view, understanding the antigen-specific immune responses in these diseases provides the key to future progress. In this respect, the investigation of GD and HT has some advantages over the study of many other autoimmune diseases. The tools are becoming available to study naturally processed and presented TPO and TSHR peptides to T cells. The role of B cells and autoantibodies in this process, such as by influencing presentation of certain peptides, is likely to be a fertile area of investigation and may explain why there is no B cell epitopic spreading from the TPO immunodominant region and why functional TSHR autoantibodies arise in GD. For this reason, obtaining and characterizing human TSHR monoclonal autoantibodies remains an important goal. The physical properties of the autoantigens may also provide insight into the autoimmune response. For example, could shedding of the TSHR ectodomain and uptake by draining lymph nodes contribute to the initiation or progression of GD? It is noteworthy that an autoimmune response does not arise to the noncleaving and nonshedding, but closely related, gonadotropin receptors. What are the precise epitopes of TSAb, TBAb, and nonfunctional TSHR autoantibodies, and what are the three-dimensional structures of autoantigens, especially when complexed with autoantibodies? Answers to these fundamental questions could lead to approaches for deviating the immune system away from strategic epitopes.

\section{Acknowledgments}

Some of the information described in this review was obtained through the support of NIH grants DK19289, DK-36182, and DK-54684.

1. Ludgate, M., Costagliola, S., and Vassart, G. 2000. Animal models of Graves' disease. In Graves' disease: pathogenesis and treatment. B. Rapoport and S.M. McLachlan, editors. Kluwer Academic Publishers. Norwell, Massachusetts, USA. 127-138.

2. McLachlan, S.M., and Rapoport, B. 1992. The molecular biology of thyroid peroxidase: cloning, expression and role as autoantigen in autoimmune thyroid disease. Endocr. Rev. 13:192-206.

3. Rapoport, B., Chazenbalk, G.D., Jaume, J.C., and McLachlan, S.M. 1998. The thyrotropin receptor: interaction with thyrotropin and autoantibodies. Endocr. Rev. 19:673-716.

4. Dai, G., Levy, O., and Carrasco, N. 1996. Cloning and characterization of the thyroid iodide transporter. Nature. 379:458-460.

5.Zeng, J., and Fenna, R.E. 1992. X-ray crystal structure of canine 
myeloperoxidase at 3 A resolution. J. Mol. Biol. 226:185-207.

6. Buckland, P.R., Rickards, C.R., Howells, R.D., Jones, E.D., and Rees Smith, B. 1982. Photo-affinity labelling of the thyrotropin receptor. FEBS Lett. 145:245-249.

7. Misrahi, M., et al. 1994. Processing of the precursors of the human thyroid-stimulating hormone receptor in various eukaryotic cells (human thyrocytes, transfected L cells and baculovirus-infected insect cells). Eur. J. Biochem. 222:711-719.

8. Chazenbalk, G.D., et al. 1997. Evidence that the thyrotropin receptor ectodomain contains not one, but two, cleavage sites. Endocrinology. 138:2893-2899.

9. Couet, J., et al. 1996. Shedding of human thyrotropin receptor ectodomain: involvement of a matrix metalloprotease. J. Biol. Chem. 271:4545-4552.

10. Chazenbalk, G.D., Jaume, J.C., McLachlan, S.M., and Rapoport, B. 1997. Engineering the human thyrotropin receptor ectodomain from a nonsecreted form to a secreted, highly immunoreactive glycoprotein that neutralizes autoantibodies in Graves' patients' sera. J. Biol. Chem. 272:18959-18965.

11. Kajava, A.V., Vassart, G., and Wodak, S.J. 1995. Modeling of the threedimensional structure of proteins with the typical leucine-rich repeats. Structure. 3:867-877.

12. McLachlan, S.M., and Rapoport, B. 2000. Autoimmune response to the thyroid in humans. Thyroid peroxidase: the common autoantigenic denominator. Int. Rev. Immunol. 19:587-618.

13. McIntosh, R.S., and Weetman, A.P. 1997. Molecular analysis of the antibody response to thyroglobulin and thyroid peroxidase. Thyroid. 7:471-487.

14. McLachlan, S.M., McGregor, A., Rees Smith, B., and Hall, R. 1979. Thyroid-autoantibody synthesis by Hashimoto thyroid lymphocytes. Lancet. 1:162-163.

15. McLachlan, S.M., and Rapoport, B. 1996. Monoclonal human autoantibodies to the TSH receptor: the holy grail and why are we looking for it? J. Clin. Endocrinol. Metab. 81:3152-3154.

16. Ruf, J., et al. 1989. Relationship between immunological structure and biochemical properties of human thyroid peroxidase. Endocrinology. 125:1211-1218.

17. Chazenbalk, G.D., et al. 1993. Human organ-specific autoimmune disease: molecular cloning and expression of an autoantibody gene repertoire for a major autoantigen reveals an antigenic dominant region and restricted immunoglobulin gene usage in the target organ. J. Clin. Invest. 92:62-74.

18. Nishikawa, T., Costante, G., Prummel, M.F., McLachlan, S.M., and Rapoport, B. 1994. Recombinant thyroid peroxidase autoantibodies can be used for epitopic "fingerprinting" of thyroid peroxidase autoantibodies in the sera of individual patients. J. Clin. Endocrinol. Metab. 78:944-949.

19. Guo, J., Yan, X.-M., McLachlan, S.M., and Rapoport, B. 2001. Search for the autoantibody immunodominant region on thyroid peroxidase: epitopic footprinting with a human monoclonal autoantibody locates a facet on the native antigen containing a highly conformational epitope. J. Immunol. 166:1327-1333.

20. Chacko, S., Padlan, E., Portolano, S., McLachlan, S.M., and Rapoport, B. 1996. Structural studies of human autoantibodies. Crystal structure of a thyroid peroxidase autoantibody Fab. J. Biol. Chem. 271:12191-12198.

21. Nagayama, Y., Wadsworth, H.L., Russo, D., Chazenbalk, G.D., and Rapoport, B. 1991. Binding domains of stimulatory and inhibitory thy- rotropin (TSH) receptor autoantibodies determined with chimeric TSHlutropin/chorionic gonadotropin receptors. J. Clin. Invest. 88:336-340.

22. Rapoport, B., McLachlan, S.M., Kakinuma, A., and Chazenbalk, G.D. 1996. Critical relationship between autoantibody recognition and TSH receptor maturation as reflected in the acquisition of mature carbohydrate. J. Clin. Endocrinol. Metab. 81:2525-2533.

23. Tahara, K., Ban, T., Minegishi, T., and Kohn, L.D. 1991. Immunoglobulins from Graves' disease patients interact with different sites on TSH receptor/LH-CG receptor chimeras than either TSH or immunoglobulins from idiopathic myxedema patients. Biochem. Biophys. Res. Commun. 179:70-77.

24. Quaratino, S., Feldmann, M., Dayan, C.M., Acuto, O., and Londei, M. 1996. Human self-reactive T cell clones expressing identical T cell receptor beta chains differ in their ability to recognize a cryptic self-epitope. J. Exp. Med. 183:349-358.

25. Dayan, C.M., et al. 1991. Autoantigen recognition by thyroid-infiltrating T cells in Graves disease. Proc. Natl. Acad. Sci. USA. 88:7415-7419.

26. Mullins, R.J., et al. 1995. Identification of thyroid stimulating hormone receptor-specific T cells in Graves' disease thyroid using autoantigentransfected Epstein-Barr virus-transformed B cell lines. J. Clin. Invest. 96:30-37.

27. Guo, J., et al. 1996. Autoantibody-mediated capture and presentation of autoantigen to $\mathrm{T}$ cells via the $\mathrm{Fc}$ epsilon receptor by a recombinant human autoantibody Fab converted to IgE. J. Immunol. Methods. 195:81-92.

28. Simitsek, P.D., Campbell, D.G., Lanzavecchia, A., Fairweather, N., and Watts, C. 1995. Modulation of antigen processing by bound antibodies can boost or suppress class II major histocompatibility complex presentation of different T cell determinants. J. Exp. Med. 181:1957-1963.

29. Serreze, D.V., et al. 1998. B lymphocytes are critical antigen-presenting cells for the initiation of $\mathrm{T}$ cell-mediated autoimmune diabetes in nonobese diabetic mice. J. Immunol. 161:3912-3918.

30. Bottazzo, G.F., Pujol-Borrell, R., Hanafusa, T., and Feldmann, M. 1983. Role of aberrant HLA-DR expression and antigen presentation in induction of endocrine autoimmunity. Lancet. 2:1115-1118.

31. Shimojo, N., et al. 1996. Induction of Graves-like disease in mice by immunization with fibroblasts transfected with the thyrotropin repector and a class II molecule. Proc. Natl. Acad. Sci. USA. 93:11074-11079.

32. Jaume, J.C., Guo, J., Wang, Y., Rapoport, B., and McLachlan, S.M. 1999. Cellular thyroid peroxidase (TPO), unlike purified TPO and adjuvant, induces antibodies in mice that resemble autoantibodies in human autoimmune thyroid disease. J. Clin. Endocrinol. Metab. 84:1651-1657.

33. Giordano, C., et al. 1997. Potential involvement of Fas and its ligand in the pathogenesis of Hashimoto's thyroiditis. Science. 275:960-963.

34. Arscott, P.L., and Baker, J.R., Jr. 1998. Apoptosis and thyroiditis. Clin. Immunol. Immunopathol. 87:207-217.

35. Tomer, Y., and Davies, T.F. 2000. The genetics of familial and non-familial hyperthyroid Graves' disease. In Graves' disease: pathogenesis and treatment. B. Rapoport and S.M. McLachlan, editors. Kluwer Academic Publishers. Norwell, Massachusetts, USA. 19-41.

36. Bahn, R.S. 2000. Pathogenesis of Graves' ophthalmopathy. In Graves' disease: pathogenesis and treatment. B. Rapoport and S.M. McLachlan, editors. Kluwer Academic Publishers. Norwell, Massachusetts, USA. 249-256.

37. Rapoport, B., Alsabeh, R., Aftergood, D., and McLachlan, S.M. 2000. Elephantiasic pretibial myxedema: insight into and a hypothesis regarding the pathogenesis of the extrathyroidal manifestations of Graves' disease. Thyroid. 10:685-692. 